\title{
Central Sensitization: Clinical Implications for Chronic Head and Neck Pain
}

\author{
Arthur S. Roberts
}

Department of Oral Medicine, Indiana University School of Dentistry, Indianapolis, 46202

\begin{abstract}
Chronic clinical pain associated with CS, is a potentially progressive, devastating, multimodal disease with a significant worldwide economic and social burden. Effective intervention is dependent upon recognizing the fundamental differences in acute and chronic pain, the effects on and by the neuromatrix upon the biopsychosocial health of the individual patient, and integrating that knowledge into a comprehensive multidisciplinary therapeutic plan.
\end{abstract}

Keywords Chronic Pain, Sensitization, Neuromatrix, Biopsychosocial, Polypharmacy

\section{Introduction}

Chronic pain is a multidimensional experience encompassing biological, psychological and social elements. Each of these elements is entangled bi-directionally with the neuromatrix as a whole. Figure 1.

There is a growing consensus that chronic pain is a disease entity. This disease has been termed maldynia by the American Medical Association. This new designation has come about in recognition that acute and chronic pain are fundamentally different experiences, demanding a fresh approach to diagnosis, therapy, and prognosis. It has been repeatedly demonstrated that many chronic pain disorders have a major component of central sensitization. Assessment and therapy for these several disorders is dependent on an understanding of central sensitization.

\section{What is Central Sensitization (CS)}

Central sensitization is thought to occur when "Sensory-afferent signals overwhelm the body's ability to filter them". $[1,2]$ When this occurs a number of pathophysiologic changes occur including neuro-immune dysfunction, neuro-endodrine dysfunction, NMDA (N-methyl-D-aspartate) dysregulation, sympatho-afferent coupling; and altered serotonin and norepinephrine production and utilization. These events are thought to occur predominantly in the mid-brain and associated structures and are influenced by elements of the neuromatrix delineated by Melzack, Woolf, and others.[1,3-5]

These pathophysiologic changes are associated with decr-

* Corresponding author:

asmr@me.com (Arthur Roberts)

Published online at http://journal.sapub.org/cmd

Copyright (C) 2011 Scientific \& Academic Publishing. All Rights Reserved eased descending inhibition, dysautonomia, and altered serotonin production/utilzation. The resulting depression, anxiety, sleep fragmentation, allodynia, and hyperalgesia characterize a number of chronic pain disorders.[2-13]

At least two etiological pathways are posited for central sensitization. The first is chronification of nociceptive pain; and involves neuroplastic changes, and peripheral sensitization as precursors to central sensitization and the resulting clinical pain. A more psychologically centered pathway is offered as an alternative to this transformed nociception. This alternate pathway begins with elevated levels of chronic stress, and encompasses elements of anxiety, sleep fragmentation, decreased pain thresholds, and dysautonomia. It is common to find both axes actively involved in the etiology of a central sensitization syndrome. Central sensitization syndromes can be thought of as the archetypical exemplars of a chronic biopsychosocial pain disorder.[1,3,5,14-18].

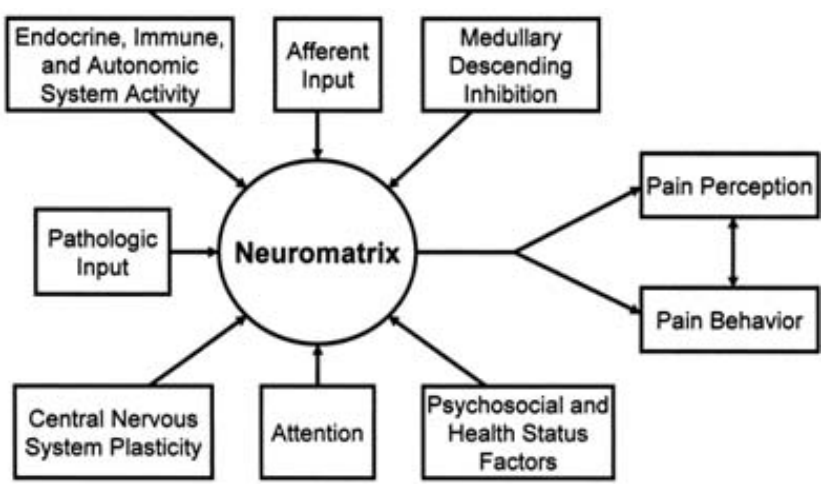

Figure 1.

\subsection{What Are Central Sensitization Syndromes (CSS)}

"A similar and overlapping group of syndromes without demonstrable pathology and bound by a common patho-physilogical mechanism of central sensitization." [2]

The balance of expert opinion holds that the following are 
part of that group: Migraine headache, tension-type headache, chronic fatigue syndrome, fibromyalgia, burning mouth syndrome, atypical odontalgia, tempormandibular disorder, and myofascial pain syndrome. In addition, there is a growing consensus that multiple chemical sensitivity, post traumatic stress disorder, depression, primary dysmenorrhea, irritable bowel syndrome, periodic limb movement disorder and restless leg syndrome are strong candidates for inclusion as central sensitization syndromes. [2, 17, 19] Figure 2.

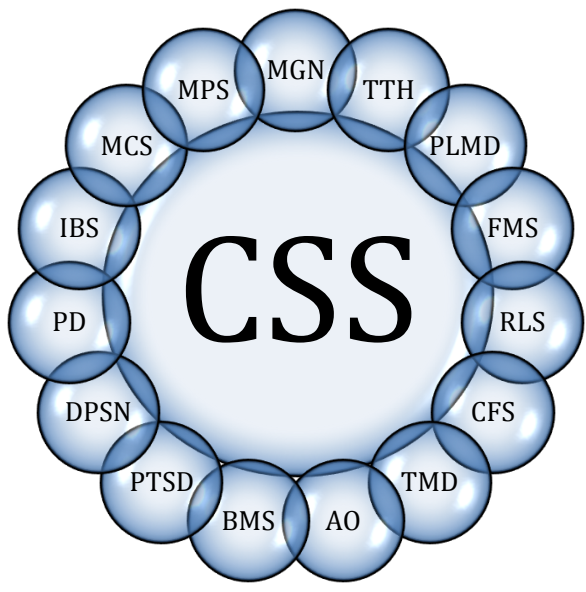

Figure 2. Adapted from Wallace and Clauw [2] MGN - Migraine, TTH Tension-type headache, IBS - Irritable bowel syndrome, CFS - Chronic Fatigue syndrome, FMS - Fibromyalgia, MPS - Myofascial pain syndrome, TMD - Tempoomandibulal dysfunction, RLS - Restless leg syndrome, PLMD - Periodic limb movement disorder, MCS - Multiple chemical sensitivity, PTSD - Post traumatic stress disorder, DPSN - Depression, PD - Primary dysmenorrhea, AO - Atypical odontalgia, BMS - Burning mouth syndrome.

\section{Diagnosis, Therapy and Prognosis}

By definition CSS are without demonstrable pathology. This leads us to clinical assessment as the most productive diagnostic approach. Allodynia, but not hyperalgesia, is an indicator of the presence of CS, as is exacerbated widespread pain highly correlated with elevated stress levels. The concurrent presentation of multiple disorders with a significant CS component is an indicator of the presence of central sensitization syndrome. [1-3]

More specifically we can assess the patient to establish the presence of characteristic sequelae of the pathophsiologic changes associated with central sensitization. These are posited to be:

- Vagal dysregulation reduces endorphin release and alters serotonin production and utilization. This produces altered accommodation of minimally painful events, and contributes to depression. [7, 18, 20]

- Sympatho-afferent coupling of sensitized trigeminal complex leads to lowered parasympathetic drive and increased sympathetic drive. This contributes to dysfunctional sleep, and anxiety via increased norepinephrine levels.[6,21-25]

- Decreased medullary descending inhibition of nociception, This increases the effective peripheral nociceptive input, and is reflected in lowered pain thresholds, hyperalgesia, allodynia, and greater impact of peripheral sensitization. [8,11,12,15-17,23,26-32]

- Hypoactivity of the hypothalamic-pituitary-adrenal axis, and autonomic nervous system alterations, produce increased sympathetic tone, low vagal tone, and contribute to immune abnormalities. This contributes to the fatigue and malaise often associated with CSS. [5,12,14,20,21,23-25,33-38]

Utilizing the resultant depression, anxiety, hyperalgesia, allodynia, stress related pain exacerbation, fatigue, and poor sleep, we can establish a list of indicators for central sensitization. Taken alone, with the possible exception of allodynia and stress related pain, none of these are pathognomonic. However, in concert they are highly suggestive of CS. In addition, when seen in association with other disorders thought to have a significant CS component, the probability of a CSS diagnosis is further increased.[1-3,5,13,14,39]

Stress, a major contributing etiologic factor in CS can be assessed using a number of non-invasive methodologies:

- HPA axis - salivary cortisol

- Autonomic nervous system - salivary alpha-amylase and heart rate variability

- Neural and immune profiles - cutaneous sweat patch

\subsection{Therapy}

Polypharmacy is one of the problems attendant to CSS therapy, and is the result of approaching each of the varied presentations of CSS as a separate and distinct disease. Each complaint not intuitively associated with CS, by either patient or provider, may result in treatment by a number of different prescribing specialists. This inevitably leads to polypharmacy, and iatrogenic contribution to decreased patient quality of life, secondary to the side effects of the multiple medications. A part of this issue may derive from failing to differentiate chronic from acute pain. While the acute symptoms of CSS disorders need to be addressed, it is essential to treat the pathways in chronic pain disease. The pathways are few, and have fairly specific therapeutic protocols associated with them. [2,5,7,8,13-15,17,19,20,32]

There are two distinctly different approaches to CSS therapy. The first, and historically more common, is to address the effects of CS after it has occurred. The second is to interrupt the CS and let the body's homeostatic mechanisms clear residual pathologic products. Optimal outcomes often depend on doing both. Within these two broad categories we have both pharmacological and non-pharmacological therapeutic options. $[1,4,6,8,13,14$, 40-44]

\subsubsection{Pharmacological Approaches}

Central sensitization is entangled with neuroplasticity and peripheral sensitization in many chronic pain syndromes, especially those deriving from transformed nociceptive pain.[1,2,13] Use of anti-inflammatory drugs 
may reduce levels of peripheral stimulus, but do not directly treat the central sensitization.[13]

Many of the drugs that we use to address central sensitization will have a beneficial effect on the ability of the patient to cope with the pain, as well as to diminish the central sensitization itself.[1,2,8,9,13,41,45]

Drugs used to address the effects of central sensitization:

- Acetaminophen

- Serotonin (SSRI) and norepinephrine (SNRI) reuptake inhibitors and tricyclic antidepressants (TCA)

- Opioids and Tramadol

Drugs that may treat the central sensitization itself:

- N-methyl-D-aspartate (NMDA) receptor blockers

- Calcium channel alpha(2) ligands

Acetaminophen

Acetaminiphen acts on the periaquaductal gray, and thence serotonergic and noradrenergic neurons to increase descending inhibition.[13,32,43,44,46,47]

Serotonin- and Norepinephrine-Reuptake Inhibitors

Serotonin re-uptake inhibitors (SSRI) and the serotonin precursor tryptophan enhance descending inhibition and are of use in stress-induced hyperalgesia.

Serotonin and norepinephrine inhibitors (SNRI) and tricyclic anti-depressants (TCA) have a dual action, affecting both spinal activity and descending inhibition via monoamine pathways in the brain. The overall effect of these drugs is to enhance sleep and coping; reduce anxiety, and catastrophizing, all of which impact upon the neuromatix and ameliorate the pain experience. $[5,13,42,43,48]$

In addition, TCAs are also thought to have a beneficial neuro-hormonal impact, and potentiate endogenous opioids. [42]

\section{Opioids and Tramadol}

The use of opioids for chronic pain is open to some controversy. From one perspective, opioids have the capacity to target critical mechanisms of central sensitization. Unfortunately, they not only target pain mechanisms, but also have significant impact upon immune function (as does central sensitization), and contribute to interleukin-1 mediated inflammatory pain hypersensitivity.[43,49-51] Taken with the significant positive reinforcement of these drugs on the reward centers of the brain, use of opioid therapy for clinical pain remains a case-by-case judgment in carefully selected and well-monitored patients.[44,52]

Tramadol, an opioid-like drug, is of interest because of its multi-modal action. Though its binding at $m u$ receptor cites is low, it is also a reuptake inhibitor of both serotonin and norepinephrine.[42]

\section{N-methyl-D-Aspartate Receptor Blockers}

Activation of NMDA-receptors (and protein kinase C) will induce hyperalgesia, supraspinal facilitatory loops, and apoptosis in the dorsal horn.

NMDA blockers (ketamine, memantine and dextromethorphan) have been applied to interrupt this process, and may be analgesic in some circumstances, however they have a fairly narrow therapeutic window.[53-55]

\section{Calcium Channel Alpha(2)Delta Ligands}

Voltage-sensitive Calcium channels at the junction of primary afferent and second order sensory neurons will sustain an enhanced release of neurotransmitters in chronic pain. Gabapentin and pregabalin interrupt this action, and reduce the release of glutamate, norepinephrine and substance $\mathrm{P}$, leading to reduction of both pre-, and postsynaptic noxious transmission. This, in turn, reduces the noxious input to the sensitized medulla.

It would follow that in those cases of stress induced central sensitization, without a nociceptive component, calcium channel ligands may be less efficacious.

Clinically it is common for these patients to cease responding to this class of drugs. Interestingly, there is some evidence that nociceptive inflammatory pain that is resistant to gabapentin, may respond to its second-generation analogue, pregabalin. Though working through somewhat different pathways some clinicians have recommended the use of carbamazepine, and its second-generation analogue oxcarbazepine, in these resistant cases. Evidence is limited for these substitutions.[42,47,54]

\subsubsection{Non-pharmacological Approaches}

Each influence upon the neuromatrix offers an opportunity to target therapeutic interventions. While some of these targets are best addressed pharmacologically, a large number of opportunities are available for non-pharmacological therapies (NPT).

For clinical purposes these NPT, like the pharmacological interventions, can be divided into two broad operative groups: Reducing CS itself; or responding to the effects of CS. In practice some of these interventions may well do both, though to varying degree.

Repetitive Transcranial Magnetic Stimulation (rTMS)

This relatively safe and non-invasive technique involves stimulation of the motor cortex and prefrontal cortex. It has seen limited application due to the short duration of its effects and significant equipment costs. It is interesting to note its greater efficacy in centrally, rather than peripherally, originated pain. In theory it reverses intra-cortical motor dysfunction, altering sensory-discriminative function, leading to restoration of descending inhibition, and also improvement of cognitive function.[56,57] However, some investigators argue that the analgesic effects are independent of descending inhibitory control and are influenced by other elements of the neuromatrix.[58]

\section{Percutaneous Electroneural Stimulation (PENS)}

Direct access to peripheral branches of multiple cranial and cervical nerves is available via percutaneous placement of electrodes on the human auricle. Utilizing a small, disposable, integrated circuit controlled, power source the patient can discreetly wear these devices for extended periods. The resulting stimulation of, primarily, the trigeminal, vagus and occipital afferents produce a number of changes in the neuromatrix. Among the observed results of PENS 
therapy are improved autonomic regulation, significant improvement in centrally mediated pain, sensory - discriminatory functions, serotonin/norepinephrine production and utilization, and endorphin production. The resultant analgesia and mood improvement appears to follow a learning curve'[59], with patient improvement being graphically additive and cumulative based upon total wearing time of the device. Most of the observed improvement is putatively ascribed to changes in the function of the trigeminal complex and anterior cingulate portions of the neuromatrix; two of the major participants of CS. As such, PENS is one of our few cost-effective, non-invasive, low co-morbidity options for the treatment of central sensitization itself, as opposed to responding to the deleterious effects of CS.[1,2,5, $6,8-10,12-15,20-23,25-28,42-44,53,55]$

The other broad category of non-pharmaceutical CS intervention encompasses manual therapy, virtual reality, improving stress tolerance, and transcutaneous electric nerve stimulation. These can be thought of as addressing the functional deficits created by the CS. In addition, some complementary and alternative approaches are being investigated and show promise, but evidence regarding their safety and efficacy is limited.

Though primarily addressing the aftermath of CS, any therapy that reduces the overall burden on the neuromatrix will reduce the self-sustaining feedback that contributes to the progressive nature of $\mathrm{CS}$.

\section{Manual Therapy}

Manual therapy improves function and additionally produces an improvement in descending inhibition, which results in widespread analgesia. However, the analgesia is of short duration and provides limited assistance in desensitizing the neuromatrix. As such, manual therapy, from a clinical perspective, is an intervention to address functional rehabilitation.[4,13,60-64]

\section{Virtual Reality}

Though there is limited evidence, virtual reality has gained some support. Its effect is thought to derive from distraction in the hyper-vigilant patient, and would thus have potential benefit in patients with movement associated nociceptive etiology. It is not in widespread use and its clinical application is currently limited.[13,65]

\section{Improving Stress Tolerance and Neuro feedback}

\section{Training}

Stress is a major etiologic and exacerbating factor for CS. Both endogenous (chronic pain) and exogenous (psychosocial changes) sources of stress can be involved. This often leads to clinical observation of the irritable, hyper-excitable chronic pain patient and is related to sympatho-afferent coupling in the hypothalamic-pituitary-adrenal axis of the neuromatrix. Additionally there are neuro-immune changes from upregulated pronociceptive immune mediators in primary afferent nociceptors. Reduction of stress levels can significantly improve the patient's pain threshold, their maladaptive behavioral responses and autonomic balance. $[12,14,17,35,36,66-68]$

\section{Transcutaneous Electrical Nerve Stimulation}

TENS is commonly employed in chronic pain states. Its effectiveness is related to activation of polysegmental inhibitory feedback, and additional elements of CS. It has some significant effect with focal, segmental chronic pain, however the results in widespread pain are equivocal.[13,40, 69-73]

\subsection{Prognosis}

CS is a potentially disabling and devastating disease whose prognosis is dependent upon the ability to identify and address the initiating and perpetuating pathways. The possibilities here fit into three classes: Those derived from transformed nociceptive pain; those derived from stress; and most commonly, those representing various combinations of the two.

In the case of transformed nociceptive pain, three elements are necessary for development of chronic clinical pain: neuroplasticity, peripheral sensitization, and central sensitization.[1] Neuroplasticity and peripheral sensitivity enable the central sensitivity. Removal, or prevention, of either or both of these, will improve the prognosis. This is best accomplished with adequate anesthesia, analgesia and inflammation control in the acute phase; early psychosocial intervention; and thorough patient education. $[2,61,62,74]$

Unfortunately, once initiated central sensitization will engender additional presentations (Figure 1.), which increase the frequency and intensity of pain, and thereby increase the endogenous stress levels. These elevated stress levels will eventually result in increased sympatho-afferent coupling, and autonomic dysfunction; leading to anxiety, poor sleep, difficulty coping, and lowered pain thresholds; and perhaps more importantly, an increased risk of developing additional presentations of CS.[2,74]

Once the CS reaches this stage, mere removal of the initiating stimulus will not insure favorable outcomes. A corollary to this: Patients with persistent biological sources of nociception, certain inflammatory diseases for instance, will have a continuing stimulus for the development and/or maintenance of CS and are likely to experience an extended disease course, including the development of additional CS presentations (syndromes), and the focus devolves to dealing with the effects of the CS, rather than control or eradication of the CS. $[2,3,74]$

In the case of stress induced CS, no biological axis may exist in the early stages. However, maladaptive behavior may engender biological issues that will contribute to maintenance and exacerbation of CS. The chronic pain associated with PTSD and depression in the apparently healthy individual may well be examples of just this sequence.

\section{Conclusions}

Chronic clinical pain associated with CS, is a potentially progressive, devastating, multimodal disease with a significant worldwide economic and social burden. Effective in- 
tervention is dependent upon recognizing the fundamental differences in acute and chronic pain, the effects on and by the neuromatrix upon the biopsychosocial health of the individual patient, and integrating that knowledge into a comprehensive multidisciplinary therapeutic plan. Its prognosis is guarded.

\section{ACKNOWLEDGEMENTS}

This paper was prepared in partial fulfilment of requirements for the MSc degree in pain management at University of Edinburgh, College of Medicine and Veterinary Medicine, Edinburgh, Scotland.

\section{REFERENCES}

[1] Woolf CJ, ed. Central Sensitization. Pain and the Control of Pain: Pain transmission, regulation and management, , ed. B. A.2009, The Biomedical \& Life Sciences Collection, Henry Stewart Talks Ltd, : London

[2] Wallace DJ, Clauw DJ, ed. Fibromyalgia and Other Central Pain Syndromes. 2005, Lippincott Williams \& Wilkins: Philidelphia

[3] Yunus MB, Inanici F, Clinical characteristics and biopathophysiological mechanisms of fibromyalgia syndrome, in Myofascial pain and fibromyalgia syndromes: a clinical guide to diagnosis and management, Baldry P, Editor 2001, Churchill Livingstone: Edinburgh

[4] Mense S, Pathophysiology of low back pain and the transition to the chronic state - experimental data and new concepts. Schmerz, 2001. 15(6): p. 413-417

[5] Melzack R, Pain and the neuromatrix in the brain. J Dent Educ, 2001. 65(12): p. 1378-1382

[6] May A, Jurgens TP, Therapeutic neuromodulation in primary headaches. Nervenarzt, 2010. Oct 24 Epub ahead of print

[7] Graven-Nielsen T, Arendt-Nielsen L, Assessment of mechanisms in localized and widespread musculoskeletal pain. Nat Rev Rheumatol, 2010

[8] Goadsby PJ, Cohen AS, Matharu MS, Trigeminal autonomic cephalgias: diagnosis and treatment. Curr Neurol Neurosci Rep, 2007. 7(2): p. 117-125

[9] Wei F, Dubner R, Zou S, Ren K, Bai G, Wei D, Guo W, Molecular depletion of descending serotonin unmasks its novel facilitory role in the development of persistent pain. $\mathrm{J}$ Neurosci, 2010. 30(25): p. 8624-8636

[10] Zhuo M, Sengupta JN, Gebhart GF, Biphasic modulation of spinal visceral nociceptive transmission from the rostroventral medial medulla in the rat. J Neurophysiol, 2002. 87: p. $2225-2236$

[11] Wulf-Johansson H, Amrutkar DV, Hay-Schmodt A, Poulsen AN, Klaerke DA, Olesen J, Jansen-Oleson I, Localization of large conductor calcium-activated potassium channels and their effect on calcitonin gene-related peptide release in the rat trigemino-neuronal pathway. Neuroscience, 2010. 167(4): p. $1091-1102$

[12] Thalakoti S, Patil VV, Damodaram S, Vause CV, Langford LE, Freeman SE, Durham PL, Neuron--glia signaling in trigeminal ganglion: implications for migraine pathology. Headache, 2008. 47(7): p. 1008-1023

[13] Nijs J, Meeus M, OsterwijckJV, Roussel N, De Kooning M, Ickmans K, Matic M, Treatment of central sensitization in patients with 'unexplained' chronic pain: what options do we have? Expert Opin Pharmacother, 2011. Epub ahead of print

[14] Meeus M, Nijs J, Central sensitization: a biopsychosocial explanation for chronic widespresd pain in patients with fibromyalgia and chronic fatigue syndrome. Clinic Rheum, 2007. 26(4): p. 465-473

[15] Bendtsen L, Central sensitization in tension-type headache: possible pathophysiological mechanisms. Cephalgia, 2000. 20(5): p. 486-508

[16] Bolay H, Reuter U, Dunn AK, Huang Z, Boas DA, Moskowitz MA, Intrinsic brain activity triggers trigeminal meningeal afferents in a migraine model. Nat Med, 2002. 8(2): p. 136-142.

[17] Dodick D, Silberstein S, Central sensitization theory of migraine: clinical implications. Headache, 2006. 46 Suppl 4: p. S182-191

[18] Graven-Nielsen T, Mense S, The peripheral apparatus of pain: evidence from animal and human studies. Clin J pain, 2001. 17(1): p. 2-10

19] Dickinson BD, Head CA, Gitlow S, Osbahr AJ, Maldynia: Pathophysiology and management of neuropathic and maladaptive pain-A report of the AMA council on science and public health. Pain Med, 2010. 11: p. 1635-1653

[20] Dorr AE, Debonnel G, Effect of vagus nerve stimulation on serotonergic and noradrenergic transmission. J Pharmcol Exp Ther, 2006. 318(2): p. 890-896

[21] Schlaepfer TE, Frick C, Zobel A, et al, Vagus nerve stimulation for depression: efficacy and safety in a European study. Psychol Med, 2008. 38(5): p. 651-661.

[22] Zhang Y, Popovic ZB, Bibevesky S, et al, Chronic vagus stimulation improves autonomic control and attenuates systemic inflammation and heart failure progression in a canine high-rate pacing model. Circ Heart Fail, 2009. 2(6): p. 692-699

[23] Multon S, Schoenen J, Pain control by vagus nerve stimulation: from animal to man.. and back Acta neurol belg, 2005(105): p. 62-67

[24] La Marca R, Nedeljkovic M, Yuan L, Maercker A, Ehlert U, Effects of auricular electrostimulation on vagal activity in healthy men: evidence from a three armed randomized trial. Clin Sci, 2010. 118: p. 537-546.

[25] George MS, N.Z., Borckardt JJ, Anderson B, Foust MJ, Burns $\mathrm{C}$, Kose $\mathrm{S}$, Short EB, Vagus nerve stimulation for the treatment of depression and other neuropsychiatric disorders. Exp Rev Neurotherapeutics, 2007. 7(1): p. 1-12

[26] Miraucourt LS, Moisset X, Dallel R, Voisin DL, Glycine inhibitory dysfunction induces a selectively dynamic, morhine-resistant, and neurokinin 1 receptor-independent mechanical allodynia. J Neurosci, 2009. 29(8): p. 2519-2527 
[27] Levy D, Jakubowski M, Burstein R, Disruption of communication between peripheral and central trigeminovascular neurons mediates the antimigraine action of $5 H T$ TB/1D recerptor agonists. Proc Natl Acad Sci USA, 2004. 23(101): p. 4274-7279

[28] Goadsby PJ, Neurostimulation in primary headache syndromes. Exp Rev Neurotherapeutics, 2007. 7(12): p. $1785-1789$

[29] Edvinsson L, Ho TW, CGRP receptor antagonism and migraine. Neurotherapeutics, 2010. 7(2): p. 164-175

[30] Ebersberger A, Pathophysiology of migraine: models to explain the generation of migraine headache. Anaesthetist, 2002. 51(8): p. 661-667

[31] Benemei S, Nicoletti P, Capone JG, Geppetti P, CGRP receptors in the control of pain and inflammation. Curr Opin Pharmacol, 2009. 9(1): p. 9-14

[32] Ackerman S, Holland PR, Lasalanda MP. Goadsby PJ, Inhibition trigeminovascular dural nociceptive afferents by $\mathrm{Ca}(2+)$-activated $\mathrm{K}(+)$ (MaxiK/BK(Ca) channel opening. Pain, 2010. 151(1): p. 128-136.

[33] Silverman M, Heim CM, Nater UM, Marques AH, Sternberg EM, Neuroendocrine and immune contributors to fatigue. PM R, 2010. 2(5): p. 338-346

[34] Ji RR, S.G., Cell signaling and the genesis of neuropathic pain. Sci STKE, 2004(252): p. 14

[35] Watkins LR, H.M., Ledeboer A, Wiesler-Frank J, Milligan ED, Maier SF, Glia as the "bad guys": implications for improving clinical pain control and the clinical utility of opioids. Brain Behav Immun, 2007. 21(2): p. 131 -146

[36] Watkins LR, H.M., Rice KC, Maier SF, the "toll" opioid-induced glial activation: improving the clinical efficacy of opioids by targeting glia. Trends Pharmacol Sci, 2009. 30(11): p. 581-591

[37] Watkins LR, M.E., Maier SF, Glial proinflammatory cytokines mediate exaggerated pain states: implications for clinical pain. Adv Exp Med Biol, 2003(521): p. 1-21

[38] Watkins LR, M.S., The pain of being sick: implications of immune to brain communication for understanding pain. Ann Rev Psychol, 2000. 51: p. 29-57

[39] Turk DC, A diathesis-stress model of chronic pain and disability following traumatic injury. Pain, 2002. Research And Management (7): p. 8-19

[40] Dufour N, Thamsborg G Oefeldt A Lundsgaard C Stender S, Treatment of Chronic Low Back Pain: A Randomized, Clinical Trial Comparing Group-Based Multidisciplinary Biopsychosocial Rehabilitation and Intensive Individual Therapist-Assisted Back Muscle Strengthening Exercises. Spine, 2010

[41] Fitzcharles MA, Lussier D, Shir Y, Management of chronic arthritis pain in the elderly. Drugs Aging, 2010. 27(6): p. 471-490

[42] Goldenberg DL, Pharmacological treatment of fibromyalgia and other chronic musculoskeletal pain. Best Pract Res Clin Rheumatol, 2007. 21: p. 499-511

[43] Millan MJ, Descending control of pain. Prog Neurobiol, 2002. 66: p. 355-374
[44] Portenoy RK, Current pharmacotherapy of chronic pain. J Pain Sympt Manag, 2000. 19(Suppl): p. 16-20

[45] Yamada K, Moriwaki K, Oiso H, Ishigooka J, High prevalence of comorbidity of migraine in outpatients with panic disorder and effectiveness of psychopharmacotherapy for both disorders: a retrospective study Psyychiatry Res, 2010. May 21 (Epub ahead of print)

[46] Smith HS, Potential analgesic mechanisms of acetaminophen. Pain Physician, 2009. 12(1): p. 269-280

[47] Suarez-Roca H, Leal L, Silva JA, et al, Reduced Neurotransmission underlies hyperalgesia induced by repeated forced swimming stress Behav Brain Res, 2008. 189: p. $159-169$

[48] Quintero L, Montero M, Avila C, et al, Long-lasting delayed hyperalgesia afterr subchronic swimm stress. Pharmacol Biochem Behav, 2000. 67: p. 449-458

[49] Samad TA, Moore KA, Sapirstein A, et al, Interleukin-1 beta mediated induction of $\mathrm{COX}-2$ in the CNS contributes to inflammatory pain hypersensitivity. Nature, 2001. 410: p. $471-475$

[50] Bazan NG, COX-2 as a multifunctional neuronal modulator. Nat Med, 2001. 7: p. 414-415

[51] Watkins LR, Maier SF, Implications of immune-to-brain communication for sickness and pain. Proc Natl Acad Sci USA, 1999. 96: p. 7710-7713

[52] Crofford LJ, Adverse effects of chronic opioid therapy for chronic musculoskeletal pain. Nat Rev Rheumatol, 2010. 6: p. 191-197

[53] Sang CN, NMDA-receptors antagonists in neuropathic pain: Experimental methods to clinical trials. J Pain Symptom Manage, 2000. 19(Suppl): p. 21-25

[54] Chiechio S, Zammataro M, Caraci F, et al, Pregabalin in the treatment of chronic pain: An overview. Clin Drug Investig, 2009. 29: p. 203-213

[55] Vanderah TW, Ossipov MH, Lai J, et al, Descending facilitation and spinal dynorphin. Pain, 2001. 92: p. 5-9

[56] Leo RJ, Latif T, Repetitive transcranial magnetic stimulation (rTMS) in experimentally induced and chronic neuropathic pain: a review. J Pain, 2007. 8: p. 453-459

[57] Lefaucheur JP, The use of repetitive transcranial magnetic stimulation (rTMS) in chronic neuropathic pain. Clin Neurophysiol, 2006. 36: p. 117-124

[58] Nahmias F, Debes C, Ciampi de Andrade D, et al, Diffuse analgesic effects of unilateral repetitive transcranial magnetic stimulation (rTMS) in healthy volunteers. Pain, 2009. 147: p. 224-232.

[59] Ji R-R, Kohno T, Moore KA, Woolf CJ, Central sensitization and LTP: do pain and memory share similar mechanisms? Trends in Neurosci, 2001. 26(12): p. 696-705

[60] Sterling M, Pedler A, Chan C, et al, Cervical lateral glide increases nociceptive flexion reflex threshold but not pressure or thermal pain thresholds in chronic whiplash associated disorders: a pilot randomised controlled trial. Man Ther, 2009. 15(2): p. 149-153

[61] Stanton-Hicks M, Baron R, Boas R, Gordh T, Harden N, 
Hendler N, Koltzenburg M, Raj P, Wilder R, Complex Regional Pain Syndromes: Guidelines for Therapy (Consensus Report). Clin J Pain, 1998. 14(2): p. 155-166

[62] Flor, H.H., C., Psychosocial Aspects of Pain: A Handbook for Health Care Providers, Progress in Pain Research and Management. Vol. 27. 2004, Seattle WA: IASP

[63] Turk DC, Flor H, Chronic pain: a biobehavaioral perrspective, in Psychosocial factors in pain: critical perspectives, Gatchel RJ, Turk DC, Editor 1999, Guilford Publications: New York. p. 18-34

[64] Thacker M, M.G., Flor H., Neuropathic pain. Management is more than pills. BMJ, 2009. 339(1)

[65] Shahrbanian S, Ma X, Korner-Bitensky N, Simmonds MJ, Scientific evidence for the effectiveness of virtual reality for pain reduction in adults with acute or chronic pain. Stud Health Technol Inform, 2009. 144: p. 40-43

[66] !!! INVALID CITATION !!!

[67] DeLeo, J.A., Basic Science of Pain. J Bone Joint Surg Am, 2006. 88(suppl_2): p. 58-62

[68] Wang H, Guo W, Yang K, Wei F, Dubner R Ren K, Contribution of primary afferent input to trigeminal astroglial hyperactivity, cytokine induction and NMDA receptor phosporylation. Open Pain J, 2010. 3: p. 144-152

[69] Wright A, Sluka KA, Nonpharmacological treatments for musculoskeletal pain. Clin J Pain, 2001. 17: p. 43-46

[70] Weiner DK, Ernst E, Complementary and alternative approaches to the treatment of persistent musculoskeletal pain. Clin J Pain, 2004. 20: p. 244-255

[71] Sauer K, Kemper C, Glaeske G, Fibromyalgia syndrome: Prevalence, pharmacological and non-pharmacological interventions in outpatient healthcare. an analysis of statutory health insurance data. Joint Bone Spine, 2011. 78(1): p. $80-84$

[72] Ghoname EA, C.W., White PF, Ahmed HE, Hamza MA, Noe $\mathrm{CE}$, Percutaneous electrical nerve stimulation: an alternative to TENS in the management of sciatica. Pain, 1999. 83: p. 193-199

[73] Branfort G, Nilsson N, Haas M, Evvans RL, Goldsmith CH, Assendelft WJJ, Bouter LM, Non-invasive physical treatments for chronic/recurrent headache (review). Cochrane Library, 2009(1)

[74] Fishman SM, Ballantyne JC, Rathwell JP, ed. Bonica's Management of Pain. 4 ed. 2009, Lippincott Williams \& Wilkins: Philadelphia 